\title{
Comparative N-Linked Glycan Analysis of Wild-Type and $\alpha 1,3-$ Galactosyltransferase Gene Knock-Out Pig Fibroblasts Using Mass Spectrometry Approaches
}

\author{
Hae-Min Park', Yoon-Woo Kim ${ }^{1}$, Kyoung-Jin Kim', Young June Kim ${ }^{3}$, Yung-Hun Yang ${ }^{4}$, Jang Mi Jin ${ }^{5}$, \\ Young Hwan Kim ${ }^{5,6}$, Byung-Gee Kim ${ }^{2}$, Hosup Shim ${ }^{3, *}$, and Yun-Gon Kim ${ }^{1, *}$
}

\begin{abstract}
Carbohydrate antigens expressed on pig cells are considered to be major barriers in pig-to-human xenotransplantation. Even after $\alpha 1,3-$ galactosyltransferase gene knock-out (GalT-KO) pigs are generated, potential non-Gal antigens are still existed. However, to the best of our knowledge there is no extensive study analyzing $\mathrm{N}$-glycans expressed on the GalT-KO pig tissues or cells. Here, we identified and quantified totally $47 \mathrm{~N}$-glycans from wild-type (WT) and GalT-KO pig fibroblasts using mass spectrometry. First, our results confirmed the absence of galactose-alpha-1,3galactose ( $\alpha$-Gal) residue in the GalT-KO pig cells. Interestingly, we showed that the level of overall fucosylated $\mathrm{N}$ glycans from GalT-KO pig fibroblasts is much higher than from WT pig fibroblasts. Moreover, the relative quantity of the N-glycolylneuraminic acid (NeuGc) antigen is slightly higher in the GalT-KO pigs. Thus, this study will contribute to a better understanding of cellular glycan alterations on GalT-KO pigs for successful xenotransplantation.
\end{abstract}

\section{INTRODUCTION}

Glycoconjugates that cover cell surfaces play a significant role in a large number of biological processes, including cell adhesion, signal transduction, and receptor activation (Crocker and

${ }^{1}$ Department of Chemical Engineering, Soongsil University, Seoul 156-743, Korea, ${ }^{2}$ School of Chemical and Biological Engineering, Seoul National University, Seoul 151-742, Korea, ${ }^{3}$ Department of Nanobiomedical Science and BK21+ NBM Global Research Center for Regenerative Medicine Dankook University, Cheonan 330-714, Korea, ${ }^{4}$ Department of Microbial Engineering, College of Engineering, Konkuk University, Seoul 143-701, Korea, ${ }^{5}$ Division of Mass Spectrometry Research, Korea Basic Science Institute, Ochang 363-883, Korea, ${ }^{6}$ Department of Bio-Analytical Science, University of Science and Technology, Daejeon 305-764, Korea

*Correspondence: shim@dku.edu (HS); ygkim@ssu.ac.kr (YGK)

Received 2 September, 2014; revised 20 October, 2014; accepted 21 October, 2014; published online 16 December 2014

Keywords: GalT-KO pig fibroblast, mass spectrometry (MS), N-glycan, $\mathrm{N}$-glycolylneuraminic acid (NeuGc)
Feizi, 1996; Ohtsubo and Marth, 2006; Roseman, 2001). Thus, a number of studies on protein glycosylation have revealed their structure and function for the governance of in vivo physiologies and diseases (Ahn et al., 2010; Dube and Bertozzi, 2005; Marth and Grewal, 2008; Roth et al., 2010; Zhao et al., 2008). In xenotransplantation, the galactose-alpha-1,3-galactose ( $\alpha$-Gal) carbohydrate antigen expressed in pig tissues had been regarded as the most important xenoantigen that interacts with natural $\alpha$-Gal antibodies responsible for hyper acute rejection (HAR) in pig-tohuman xenotransplantation (Sandrin and McKenzie, 1994). To overcome this barrier to successful xenografts, several groups successfully produced GalT-KO pigs that do not express the $\alpha$ Gal antigens (Dai et al., 2002; Kolber-Simonds et al., 2004; Lai et al., 2002). Nonetheless, the non-Gal antigens originally present on pig cell surfaces induce complement mediated lysis and antibody dependent cellular cytotoxicity (ADCC) of pig cells by binding to non-Gal anti-pig natural antibodies in human serum (Baumann et al., 2007). In addition, it has been reported that the knockout of pig $\alpha 1,3$-galactosyltransferase gene results in alteration of the expression of carbohydrate antigens expressed on the pig cell surface (Diswall et al., 2010; Miyagawa et al., 2010; Park et al., 2011; Puga Yung et al., 2012). More recently, Lutz et al. (2013) produced double knockout pigs deficient in $\alpha$ Gal and NeuGc epitopes and Burlak et al. (2013) reported on $\mathrm{N}$-glycosylation changes in pig serum caused by knockout of

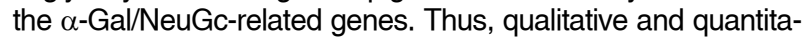
tive analysis of pig cell glycans containing non-Gal antigens is preferentially required prior to therapeutic strategies (e.g., genetic modifications) aimed at preventing xenograft rejection in xenotransplantation.

Mass spectrometric techniques have been a powerful tool for the analysis of cellular glycans, which possess great complexity and diversity in structure (Haslam et al., 2006; North et al., 2009). In contrast to the indirect method of lectin or glycanbinding antibody-based assays, MS analysis directly enables complete characterization of glycan structures. Moreover, several attempts for MS-based quantitation have been recently reported, and these have been found to have good quantitative performance when compared to high-performance liquid chromatography (HPLC) (Gil et al., 2008; 2010; Jang et al., 2009; Kang et al., 2008; Toyoda et al., 2008). Recent studies on 
GalT-KO pigs reported glycolipid analysis of pig tissues and cells using an LC-MS instrument, demonstrating that such a tool allowed researchers to characterize the glycolipid-derived glycan structures (Diswall et al., 2010; Puga Yung et al., 2012). However, the LC-MS and lectin immunostaining methods used in previous studies are limited because they cannot provide reliable quantitative information regarding the individual glycans present on pig cells and tissues.

In this study, we report on the identification of $\mathrm{N}$-glycans derived from WT and GalT-KO pig fibroblasts. We also provide a quantitative comparison of the $\mathrm{N}$-glycosylation profiles between these pig cells using MS-based approaches. MS combined with solid-phase permethylation enable both quantitative and qualitative analysis of cellular glycans. We identified diverse $N$ glycans, including high-mannose type and complex type glycans on pig fibroblasts. In particular, we found that the relative quantity of sialic acid-containing glycans increased in GalT$\mathrm{KO}$, and the amount of NeuGc, considered to be a promising non-Gal antigen, was slightly higher in GalT-KO than in WT. This qualitative and quantitative information is expected to be valuable for successful xenotransplantation.

\section{MATERIALS AND METHODS}

\section{Chemicals and reagents}

Sodium hydroxide (beads, 20-40 mesh), 5-dihydroxybenzoic acid (DHB), dimethyl sulfoxide (DMSO), methyl iodide, and trifluoroacetic acid (TFA) were purchased from Sigma-Aldrich (USA). Peptide $\mathrm{N}$-glycosidase $\mathrm{F}$ (PNGase F) was obtained from Roche (Germany). Porous graphitic carbon (PGC) cartridge was purchased from Thermo Scientific (USA) and Microspin column was obtained from Harvard Apparatus (USA). Acetic acid, acetonitrile (ACN), and chloroform were purchased from Junsei (Japan). All aqueous solutions were prepared with ReadyPrep ${ }^{\text {TM }}$ Proteomic Grade Water (Bio-Rad, USA)

\section{Isolation of homozygous GalT-KO cells}

The Institutional Animal Care and Use Committee of the Dankook University approved all animal procedures. Fibroblasts were cultured from ear skin biopsies of the heterozygous GalT$\mathrm{KO}$ piglets as previously reported (Ahn et al., 2011). Selection of homozygous GalT-KO cells derived from the loss of heterozygosity was performed as described elsewhere (Fujimura et al., 2008). Briefly, $5 \times 10^{5}$ heterozygous GalT-KO cells were washed twice with Dulbecco's phosphate buffered saline (DPBS), resuspended with $1 \mathrm{ml}$ of DPBS containing $4 \mu \mathrm{g}$ biotinconjugated IB4-lectin (EY Laboratories, USA), a lectin that specifically binds to Gal $1,3 \mathrm{Gal}$ epitopes, in a $1.5-\mathrm{ml}$ tube, and incubated for $1 \mathrm{~h}$ on ice while tapping the tube to prevent precipitation of the cells. After washing cells twice with DPBS by centrifugation $4 \mathrm{mg}$ of Dynabeads M-280 Streptavidin (Invitrogen, USA) was added to the pellet, and the resulting mixture was again incubated with the IB4-lectin treatment. Then, the tube was placed on a magnet for 2-3 min, and the supernatant was transferred to a new tube on a magnet. This procedure was repeated three times. The final supernatant was cultured in Dulbecco's modified Eagle medium (DMEM) containing 10\% (v/v) fetal bovine serum (FBS) in $60-\mathrm{mm}$ culture dish for $10-14$ days until colonies reached approximately $2 \mathrm{~mm}$ in diameter. The culture dish containing fibroblast colonies was washed twice with DPBS and covered with dimethylpolysiloxane (DMPS). Colonies were dissociated by injecting $30 \mu \mathrm{l}$ of $0.25 \%$ trypsin-EDTA on top of each colony underneath DMPS using a micropipette. After incubation for $5 \mathrm{~min}$ at $39^{\circ} \mathrm{C}$, fibroblasts dissociated from each colony were trans- ferred onto $0.1 \%$ gelatin-coated 24 -well culture dish for clonal culture. Clones of homozygous GalT-KO were analyzed by PCR as previously reported (Ahn et al., 2011). Briefly, genomic DNA was prepared from fibroblasts using DNeasy Tissue Kit (Qiagen, USA) according to the manufacturer's protocol. Using Maxime PCR premix kit (Intron Biotechnology, Korea) in a $20 \mu$ reaction volume, amplification of the target gene was carried out using forward 5'-AGAGGTCGTGACCATAACCAGAT-3' and reverse 5'-AGCCC-ATGCTGAACATCAAGTC-3' primers. Conditions for PCR were as follows: 30 cycles of $15 \mathrm{~s}$ at $94^{\circ} \mathrm{C}, 30 \mathrm{~s}$ at $65^{\circ} \mathrm{C}, 10$ $\mathrm{min}+20 \mathrm{~s}$ increase/cycle at $68^{\circ} \mathrm{C}$; and one final $7 \mathrm{~min}$ cycle at $68^{\circ} \mathrm{C}$. Amplification products $(9.2 \mathrm{~kb}$ GalT gene-targeted and/or $7.4 \mathrm{~kb}$ wild type allele) were analyzed by $0.4 \%$ agarose gel electrophoresis. Fibroblasts confirmed to be GalT-KO were used for subsequent experiments.

\section{Membrane fractionation from pig fibroblasts}

Cell membrane fractionation was performed following descriptions in a previous study (Kim et al., 2009a), with minor modifications. Briefly, pig fibroblasts (WT and GalT-KO, respectively) consisting of $1 \times 10^{6} \sim 1 \times 10^{7}$ cells were washed twice with $1 \times$ phosphate buffered saline (PBS) by centrifugation at $90 \times g$ for 5 min. The washed cell pellets were homogenized by sonication followed by centrifugation at 3,500 rpm (Hanil Research \& Development Mircro17TR, Korea) for 10 min to remove cell debris and the nuclear fraction. The supernatant was centrifuged at 100,000 $\times g$ for $1 \mathrm{~h}$ and then precipitates (membrane fraction) were washed with $1 \times$ PBS three times to remove the cytosol fraction. The washed precipitates were dissolved in $50 \mathrm{mM}$ sodium phosphate buffer $(\mathrm{pH} 7.5)$ for further enzyme reaction.

\section{Deglycosylation of membrane fractions}

The membrane proteins were incubated at $95^{\circ} \mathrm{C}$ for $3 \mathrm{~min}$ for denaturation. The denatured proteins were added to $5 \mathrm{U}$ of PNGase $\mathrm{F}$ and incubated at $37^{\circ} \mathrm{C}$ for $16 \mathrm{~h}$ for deglycosylation. The sample was added to a 4-fold volume of ethanol and incubated at $-20^{\circ} \mathrm{C}$ for $2 \mathrm{~h}$. The $\mathrm{N}$-deglycosylated proteins were removed by centrifugation at $13,500 \mathrm{rpm}$ for $15 \mathrm{~min}$ and supernatants containing $\mathrm{N}$-glycans were carefully transferred to new tubes and dried in a vacuum evaporator for further purification.

\section{Purification of $\mathrm{N}$-glycans}

The released $\mathrm{N}$-glycans were purified using PGC. The PGC cartridge was washed with $6 \mathrm{~mL}$ of water and $3 \mathrm{ml}$ of $50 \%(\mathrm{v} / \mathrm{v})$ ACN/water containing $0.1 \%$ TFA. $1 \mathrm{ml}$ of the sample in water was loaded on the cartridge and then washed with water and $5 \%(\mathrm{v} / \mathrm{v})$ ACN/water containing $0.1 \%$ TFA to remove salts and other contaminants. The $N$-glycans were eluted with $50 \%(\mathrm{v} / \mathrm{v})$ ACN/water containing $0.1 \%$ TFA and then completely dried for further glycan derivatization.

\section{Permethylation of $\mathrm{N}$-glycans}

The derivatization of the purified $\mathrm{N}$-glycans was performed using a solid-phase permethylation method (Kang et al., 2008). Briefly, the $\mathrm{N}$-glycans were resuspended in $141.6 \mu \mathrm{l}$ of DMSO, $52.8 \mu \mathrm{l}$ of iodomethane, and $5.6 \mu \mathrm{l}$ of water. A NaOH-packed spin column was washed with ACN and DMSO and then the sample solution was loaded on the spin column followed by centrifugation at 1,200 rpm for $15 \mathrm{~min}$. The step was repeated at least eight times for complete permethylation. The sample was collected and then extracted with $200 \mu$ of chloroform and $200 \mu$ of water by vortexing. The permethylated $N$-glycans in the organic solvent phase were dried prior to MS analyses. 


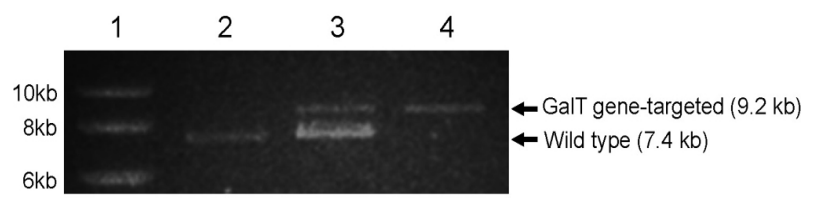

Fig. 1. PCR analysis of genomic DNA from homozygous GalT-KO cells. Lane 1, $1 \mathrm{~kb}$ ladder; lane 2, wild-type cells; lane 3, heterozygous GalT-KO cells; lane 4, homozygous GalT-KO cells.

\section{MALDI-TOF MS/MALDI-QIT-TOF MS analysis}

MS analysis was carried out using a matrix-assisted laser desorption/ionization time-of-flight (MALDI-TOF) MS instrument with an Autoflex system from Bruker Daltonics (Bruker, Germany). Tandem mass spectrometric analysis was performed using an Axima Resonance MALDI-quadrupole ion trap (QIT)-TOF instrument (Shimadzu, UK). These mass spectrometers were operated in positive-ion reflectron mode. The matrix solution used was $10 \mathrm{mg} / \mathrm{ml} \mathrm{DHB}$ in $50 \%$ methanol. Data acquisition and processing in MS and MS/MS analysis were performed with flexAnalysis 3.3 software (Bruker, Germany) and Launchpad 2.9.3 software (Kratos Analytical Ltd., UK), respectively. The mass spectrometric analysis was triplicated for all samples.

\section{RESULTS}

Fibroblasts with biallelic disruption of the GalT gene resulting from loss of heterozygosity (LOH) in heterozygous GalT-KO were obtained by selection using IB4-Dynabead. As shown in Fig. 1, PCR analysis confirmed that fibroblasts from the colony selected contained only the GalT gene-targeted allele $(9.2 \mathrm{~kb})$ without the wild type allele $(7.4 \mathrm{~kb})$, demonstrating that homozygous GalT-KO fibroblasts were successfully isolated.

In this study, we sought to identify $\mathrm{N}$-glycans on the cell surface of WT and GalT-KO pig fibroblasts using an MS-based approach. The scheme for the proposed approach of this study is described in Fig. 2. Cell membrane proteins were fractionat- ed from pig fibroblast lysate via ultracentrifugation, and then $N$ glycans were released from the cell membrane proteins by peptide $\mathrm{N}$-glycosidase $\mathrm{F}$ (PNGase $\mathrm{F}$ ) digestion. Next, the released $\mathrm{N}$-glycans were desalted using porous graphitic carbon (PGC) cartridges for the solid-phase extraction. Finally, the purified $\mathrm{N}$-glycans were permethylated and subsequently analyzed via MALDI-TOF MS and MS/MS.

The representative MALDI spectra obtained from WT and GalT-KO pig fibroblasts are shown in Fig. 3 . The $N$-glycan profile showed a total of $47 \mathrm{~N}$-glycans on pig fibroblasts, including high-mannose type glycans and complex type glycans with bi-, tri-, and tetra-antennary structures (Table 1). The predicted structures were proposed according to structural information obtained in our previous studies (Kim et al., 2006; 2008). The MS results also indicated that sialylated glycans and fucosylated glycans were abundant components of the complex-type glycans. Notably, the $\alpha$-Gal antigen at an $m / z$ value of 2652.4, considered to be the major xenoantigen, was not detected in Gal-KO pig fibroblasts but was observed in trace amounts in WT pig fibroblasts. This $\alpha$-Gal xenoantigen, which was deduced to be a two $\alpha$-galactosylated bi-antennary glycan due to its molecular weight, was previously found in other pig organs (Kim et al., 2006; 2008; 2009a; 2009b). We also confirmed that $\alpha$-Gal-terminated $N$-glycan was absent in GalT-KO pig fibroblasts based on the MS profiles. On the other hand, five $\mathrm{N}$-glycans (proposed to be NeuGc-containing glycans observed at $\mathrm{m} / \mathrm{z}$ values of $2186.2,2822.5,2996.6,3445.8$, and 3806.7) were detected in both WT and GalT-KO pig fibroblasts.

To quantify the $\mathrm{N}$-glycans in pig fibroblasts, we performed a MALDI-TOF MS-based quantitative analysis using solid-phase permethylation (Kang et al., 2008). The results showed that the relative quantities of sialylated $(92.3 \%)$ glycans in GalT-KO pig fibroblasts were higher than those in WT (69.9\%) (Fig. 4a). Most notably, a sialylated glycan composed of $\mathrm{Hex}_{5} \mathrm{HexNAc}_{4} \mathrm{Fuc}_{1}$ $\mathrm{NeuAC}_{2}$ was dramatically elevated in the GalT-KO (14.2\%) fibroblasts relative to the WT fibroblasts (1.5\%). In addition, $\mathrm{Hex}_{5}$ $\mathrm{HexNAc}_{4} \mathrm{Fuc}_{1} \mathrm{NeuAc}_{1}$ and $\mathrm{Hex}_{6} \mathrm{HexNAc}_{5} \mathrm{Fuc}_{1} \mathrm{NeuAc}_{2-3}$ (2.5\% and $2.9 \%$, respectively) glycans were only observed or were in-

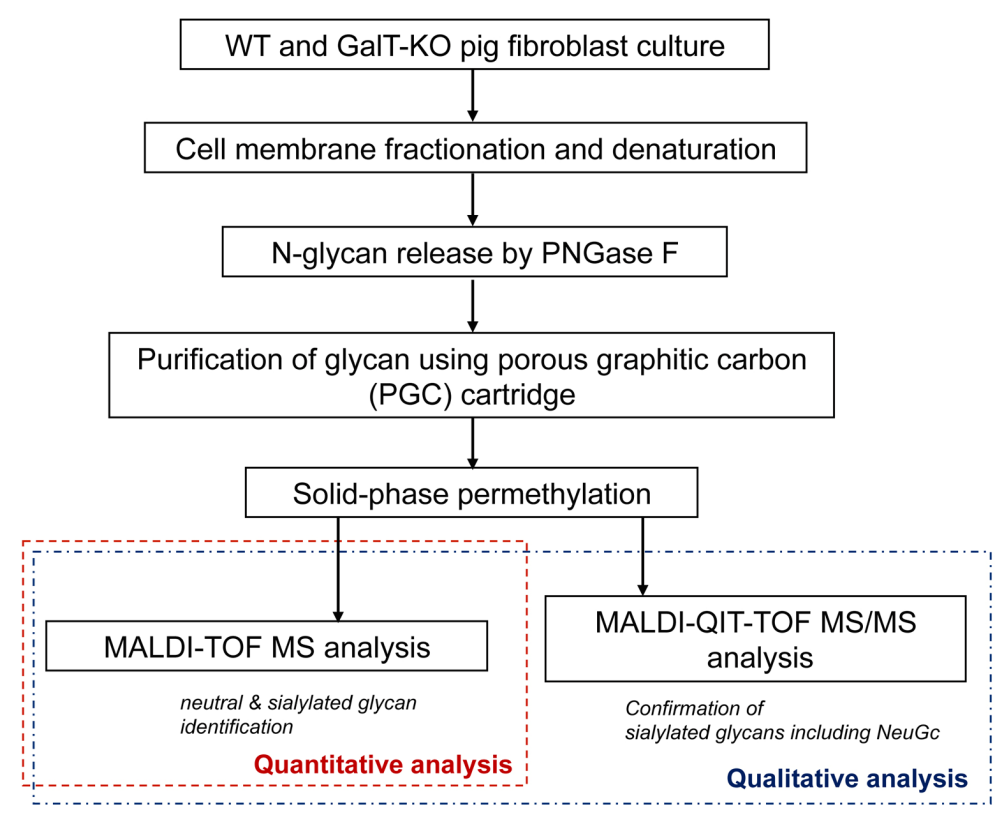

Fig. 2. Overall strategy for $\mathrm{N}$-glycan analysis of the pig fibroblasts using mass spectrometric technologies. The purified $\mathrm{N}$-glycans from membrane fractions of the pig fibroblasts were permethylated using a solid-phase permethylation protocol. After the neutralization of sialic acids, they were analyzed by using MALDI-TOF MS and MALDI-QIT-TOF MS/MS. 


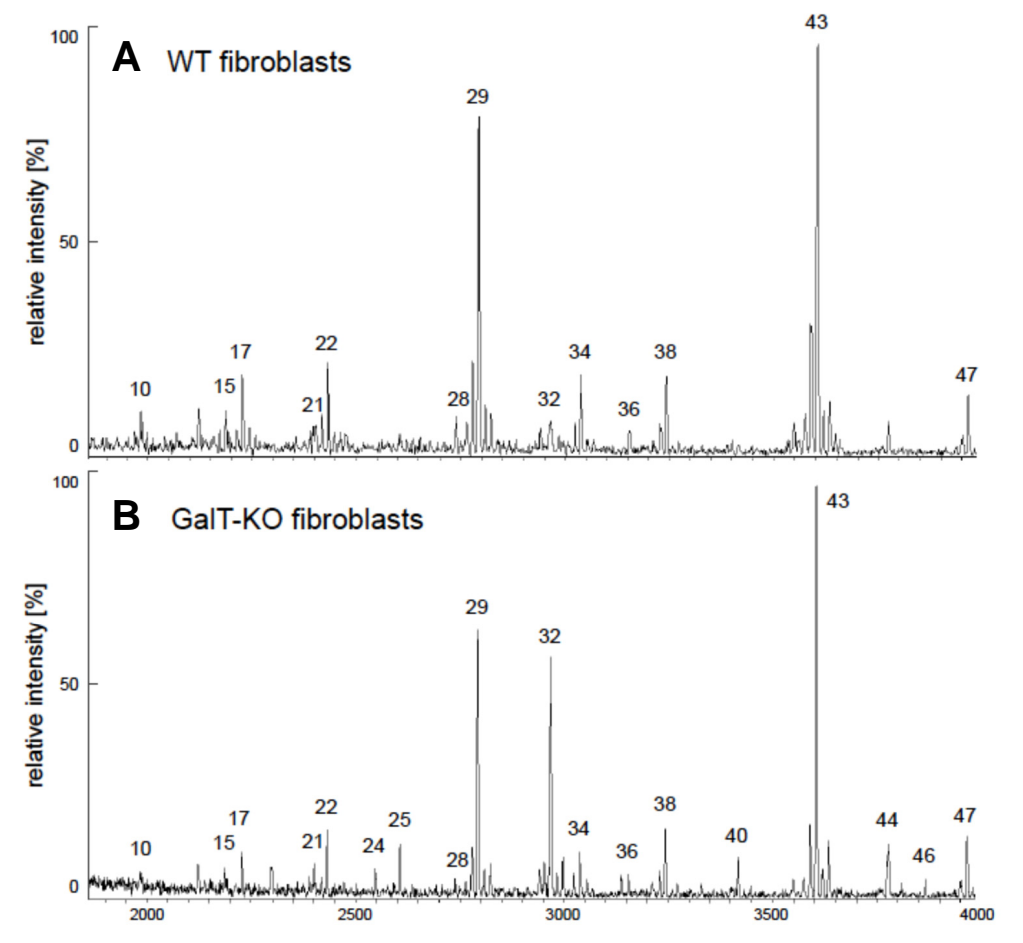

A

sialylated glycan

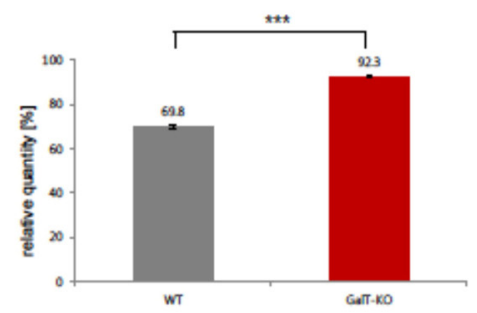

B

fucosylated glycan

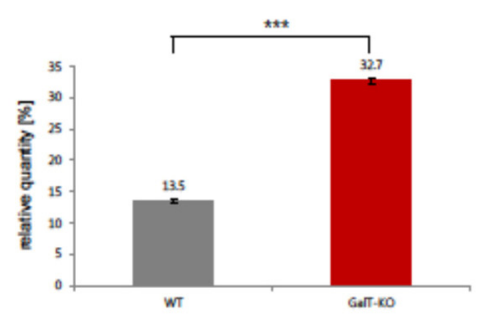

Fig. 3. Positive ion MALDI-TOF mass spectra of the $N$-glycans isolated from pig (A) wild-type and (B) GTKO fibroblasts. Major peaks detected by MALDI-TOF MS are numbered and total $\mathrm{N}$-glycans are listed in the Table 1.

Fig. 4. Relative quantitative comparison of (A) sialylated glycans, (B) fucosylated glycans, and (C) NeuGc-containing glycans. (*** $P$ value < 0.001 , * $P$ value $<0.05$; $P$ values were derived from the two-tailed Student $t$-test $n=3$ for wild type pig fibroblasts $/ \mathrm{n}=3$ for GalT-KO pig fibroblasts. Error bars show SEM).

creased in GalT-KO fibroblasts. Interestingly, we found that these increased sialylated glycans contained a core-fucose, and these glycans consequently played lead roles in the increase of overall fucosylation levels as well as in the sialylation of the pig GalT-KO, as shown in Fig 4B. We also quantified NeuGc-containing $N$ glycans, and the result showed that the quantity of NeuGc antigens was slightly higher in GalT-KO (6.1\%) compared to WT (4.5\%) (Fig. 4C).

We carried out tandem mass spectrometry analysis using MALDI-QIT TOF MS to validate the $N$-glycan structures in the pig fibroblasts. The above-mentioned Hex $\mathrm{HexNAc}_{4} \mathrm{Fuc}_{1} \mathrm{NeuAc}_{1-2}$ and $\mathrm{Hex}_{6} \mathrm{HexNAc}_{5} \mathrm{Fuc}_{1} \mathrm{NeuAc}_{2-3}$ glycans were analyzed, and the relative quantities were higher in GalT-KO pig fibroblasts than in WT fibroblasts. As shown in Fig. 5, the chemical structures of the glycans were elucidated by peak assignment of fragment ions in mass spectra and were therefore confirmed to be $\mathrm{N}$-glycans containing both sialic acid and core-fucose residues. For example, fragment ions at $\mathrm{m} / \mathrm{z}$ values of 2230.2 ,
2591.4, 3040.3, and 3401.4 were generated from parent ions at $\mathrm{m} / \mathrm{z} 2605.4,2966.6,3415.7$, and 3776.6, respectively, by the loss of an $\mathrm{N}$-acetylneuraminic acid residue (375.2 Da). The parent ions also produced a fragment ion at $\mathrm{m} / \mathrm{z} 1303.7$ or 1317.7, corresponding to a fucosylated $N$-glycan core structure. In addition, the NeuGc-containing $\mathrm{N}$-glycans $\mathrm{Hex}_{5} \mathrm{HexNAc}_{4}$

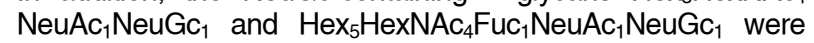
confirmed by MS/MS analysis (Fig. 6). For instance, fragment ions at $\mathrm{m} / \mathrm{z} 2417.4$ and 2591.3 were generated from parent ions at $\mathrm{m} / \mathrm{z} 2822.5$ and 2996.6 by cleavage of a NeuGc residue (405.1 Da).

\section{DISCUSSION}

In the present study, the nature of $\mathrm{N}$-glycans isolated from WT and GalT-KO pig fibroblasts was investigated. With respect to the carbohydrate antigen expressed on the surface of WT pig cells, an $\alpha$-Gal antigen and two $\alpha$-galactosylated bi-antennary 
Table 1. Identification of $N$-linked glycans derived from WT and GalT-KO pig fibroblasts

\begin{tabular}{|c|c|c|c|c|c|c|c|c|c|c|}
\hline \multirow{2}{*}{ Peak no. } & \multicolumn{2}{|c|}{$\begin{array}{c}\mathrm{M}+\mathrm{Na}]^{+} \\
m / z\end{array}$} & \multicolumn{5}{|c|}{ Compositions } & \multirow{2}{*}{ Proposed structure } & \multicolumn{2}{|c|}{$\begin{array}{c}\% \text { total } \\
\text { MALDI }^{\mathrm{a}} \\
\end{array}$} \\
\hline & $\mathrm{Cal}^{c}$ & $\operatorname{Exp}^{d}$ & Hex & HexNAc & Fuc & $\mathrm{NeuAc}$ & NeuGc & & WT & GalT-KO \\
\hline 1 & 1579.8 & 1579.9 & 5 & 2 & 0 & 0 & 0 & & $13.3( \pm 0.60)$ & $3.6( \pm 0.35)$ \\
\hline 2 & 1590.8 & 1590.9 & 3 & 3 & 1 & 0 & 0 & & $2.1( \pm 0.15)$ & $0.5( \pm 0.19)$ \\
\hline 3 & 1620.8 & 1620.9 & 4 & 3 & 0 & 0 & 0 & & $1.1( \pm 0.28)$ & trace \\
\hline 4 & 1661.8 & 1661.9 & 3 & 4 & 0 & 0 & 0 & & $0.9( \pm 0.21)$ & $0.6( \pm 0.23)$ \\
\hline 5 & 1783.9 & 1784.0 & 6 & 2 & 0 & 0 & 0 & & $3.4( \pm 0.10)$ & $0.5( \pm 0.31)$ \\
\hline 6 & 1794.9 & 1795.0 & 4 & 3 & 1 & 0 & 0 & & $0.5( \pm 0.08)$ & trace \\
\hline 7 & 1824.9 & 1825.0 & 5 & 3 & 0 & 0 & 0 & & $0.6( \pm 0.09)$ & trace \\
\hline 8 & 1835.9 & 1836.0 & 3 & 4 & 1 & 0 & 0 & & $1.6( \pm 0.15)$ & $0.4( \pm 0.18)$ \\
\hline 9 & 1865.9 & 1866.0 & 4 & 4 & 0 & 0 & 0 & $0^{-}$ & $0.4( \pm 0.12)$ & trace \\
\hline 10 & 1982.0 & 1982.1 & 4 & 3 & 0 & 1 & 0 & & $1.5( \pm 0.09)$ & $1.0( \pm 0.35)$ \\
\hline 11 & 1988.0 & 1988.1 & 7 & 2 & 0 & 0 & 0 & $0^{-}$ & $0.9( \pm 0.13)$ & $0.3( \pm 0.08)$ \\
\hline 12 & 1999.0 & 1999.1 & 5 & 3 & 1 & 0 & 0 & & $0.6( \pm 0.21)$ & $0.1( \pm 0.05)$ \\
\hline 13 & 2040.0 & 2040.1 & 4 & 4 & 1 & 0 & 0 & $0-$ & $0.4( \pm 0.05)$ & $0.2( \pm 0.02)$ \\
\hline 14 & 2070.0 & 2070.1 & 5 & 4 & 0 & 0 & 0 & $\begin{array}{l}0-90=0 \\
0=0\end{array}$ & $0.4( \pm 0.15)$ & trace \\
\hline 15 & 2186.2 & 2186.2 & 4 & 3 & 1 & 0 & 1 & & $1.9( \pm 0.03)$ & $1.2( \pm 0.18)$ \\
\hline & & & 5 & 3 & 0 & 1 & 0 & & & \\
\hline 16 & 2192.1 & 2192.2 & 8 & 2 & 0 & 0 & 0 & $\begin{array}{c}0 \\
0\end{array}$ & $0.7( \pm 0.19)$ & $0.4( \pm 0.04)$ \\
\hline
\end{tabular}


Comparative Glycomics for GalT-KO Pig Fibroblasts

Hae-Min Park et al.

Table 1. Identification of $N$-linked glycans derived from WT and GalT-KO pig fibroblasts

\begin{tabular}{|c|c|c|c|c|c|c|c|c|c|c|}
\hline \multirow{2}{*}{ Peak no. } & \multicolumn{2}{|c|}{$\begin{array}{c}{[\mathrm{M}+\mathrm{Na}]^{+}} \\
\mathrm{m} / \mathrm{z}\end{array}$} & \multicolumn{5}{|c|}{ Compositions } & \multirow{2}{*}{ Proposed structure } & \multicolumn{2}{|c|}{$\begin{array}{c}\text { \% total } \\
\text { MALDI }^{\mathrm{a}} \\
\end{array}$} \\
\hline & $\mathrm{Cal}^{\mathrm{c}}$ & $\operatorname{Exp}^{d}$ & Hex & HexNAc & Fuc & $\mathrm{NeuAc}$ & NeuGc & & WT & GalT-KO \\
\hline 17 & 2227.1 & 2227.2 & 4 & 4 & 0 & 1 & 0 & & $3.7( \pm 0.19)$ & $2.0( \pm 0.31)$ \\
\hline 18 & 2244.2 & 2244.3 & 5 & 4 & 1 & 0 & 0 & O- & $0.9( \pm 0.05)$ & $0.3( \pm 0.02)$ \\
\hline 19 & 2360.2 & 2360.3 & 5 & 3 & 1 & 1 & 0 & & trace & $0.4( \pm 0.10)$ \\
\hline 20 & 2390.2 & 2390.3 & 6 & 3 & 0 & 1 & 0 & & $0.7( \pm 0.17)$ & $0.3( \pm 0.24)$ \\
\hline 21 & 2401.2 & 2401.3 & 4 & 4 & 1 & 1 & 0 & $\diamond-$ & $1.4( \pm 0.28)$ & $1.4( \pm 0.09)$ \\
\hline 22 & 2431.2 & 2431.3 & 5 & 4 & 0 & 1 & 0 & O & $4.3( \pm 0.11)$ & $3.5( \pm 0.22)$ \\
\hline 23 & 2472.2 & 2472.4 & 4 & 5 & 0 & 1 & 0 & $\diamond-$ & $1.1( \pm 0.22)$ & $0.7( \pm 0.04)$ \\
\hline 24 & 2547.3 & 2547.4 & 5 & 3 & 0 & 2 & 0 & & $\mathrm{ND}^{\mathrm{b}}$ & $1.3( \pm 0.15)$ \\
\hline 25 & 2605.3 & 2605.4 & 5 & 4 & 1 & 1 & 0 & $\rightarrow 0$ & $0.3( \pm 0.08)$ & $2.9( \pm 0.13)$ \\
\hline 26 & 2652.3 & 2652.4 & 7 & 4 & 1 & 0 & 0 & $\begin{array}{l}0-0= \\
0-0\end{array}$ & $0.6( \pm 0.14)$ & ND \\
\hline 27 & 2676.3 & 2676.4 & 5 & 5 & 0 & 1 & 0 & $\diamond$ & $0.4( \pm 0.19)$ & $0.2( \pm 0.23)$ \\
\hline 28 & 2764.4 & 2764.4 & 6 & 6 & 0 & 0 & 0 & & $1.4( \pm 0.26)$ & $0.4( \pm 0.38)$ \\
\hline 29 & 2792.4 & 2792.4 & 5 & 4 & 0 & 2 & 0 & $\rightarrow 0-30-=$ & $17.2( \pm 0.42)$ & $15.3( \pm 0.36)$ \\
\hline 30 & 2822.4 & 2822.5 & 5 & 4 & 0 & 1 & 1 & & $1.8( \pm 0.36)$ & $1.7( \pm 0.07)$ \\
\hline 31 & 2880.4 & 2880.5 & 6 & 5 & 0 & 1 & 0 & $\checkmark \mid \begin{array}{l}0 \\
0\end{array}$ & $0.3( \pm 0.04)$ & $0.2( \pm 0.20)$ \\
\hline 32 & 2966.5 & 2966.6 & 5 & 4 & 1 & 2 & 0 & $.000=1$ & $1.5( \pm 0.10)$ & $14.3( \pm 0.46)$ \\
\hline 33 & 2996.5 & 2996.6 & 5 & 4 & 1 & 1 & 1 & $\diamond 0$ & $0.2( \pm 0.17)$ & $2.2( \pm 0.04)$ \\
\hline
\end{tabular}

(Continued) 
Table 1. Identification of $N$-linked glycans derived from WT and GalT-KO pig fibroblasts

\begin{tabular}{|c|c|c|c|c|c|c|c|c|c|c|}
\hline \multirow{2}{*}{ Peak no. } & \multicolumn{2}{|c|}{$\begin{array}{c}{[\mathrm{M}+\mathrm{Na}]^{+}} \\
\mathrm{m} / \mathrm{z}\end{array}$} & \multicolumn{5}{|c|}{ Compositions } & \multirow{2}{*}{ Proposed structure } & \multicolumn{2}{|c|}{$\begin{array}{l}\text { \% total } \\
\text { MALDI }^{\mathrm{a}}\end{array}$} \\
\hline & $\mathrm{Cal}^{\mathrm{c}}$ & $\operatorname{Exp}^{d}$ & Hex & HexNAc & Fuc & NeuAc & NeuGc & & WT & GalT-KO \\
\hline 34 & 3037.5 & 3037.6 & 5 & 5 & 0 & 2 & 0 & & $3.6( \pm 0.25)$ & $2.3( \pm 0.12)$ \\
\hline 35 & 3054.5 & 3054.5 & 6 & 5 & 1 & 1 & 0 & 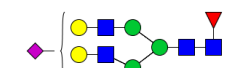 & $0.4( \pm 0.01)$ & $0.9( \pm 0.11)$ \\
\hline 36 & 3153.6 & 3153.6 & 5 & 4 & 0 & 3 & 0 & 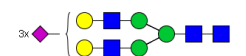 & $0.9( \pm 0.06)$ & $0.8( \pm 0.34)$ \\
\hline 37 & 3211.6 & 3211.7 & 5 & 5 & 1 & 2 & 0 & 7 & $0.3( \pm 0.11)$ & $0.4( \pm 0.38)$ \\
\hline 38 & 3241.6 & 3241.7 & 6 & 5 & 0 & 2 & 0 & 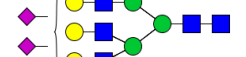 & $4.3( \pm 0.36)$ & $3.9( \pm 0.03)$ \\
\hline 39 & 3327.7 & 3327.7 & 5 & 4 & 1 & 3 & 0 & $x \bullet-\{$ & $0.1( \pm 0.09)$ & $0.4( \pm 0.00)$ \\
\hline 40 & 3415.7 & 3415.7 & 6 & 5 & 1 & 2 & 0 & & $0.1( \pm 0.21)$ & $2.5( \pm 0.49)$ \\
\hline 41 & 3445.7 & 3445.8 & 6 & 5 & 1 & 1 & 1 & & $0.2( \pm 0.05)$ & $0.2( \pm 0.16)$ \\
\hline 42 & 3503.7 & 3503.7 & 7 & 6 & 1 & 1 & 0 & & $0.1( \pm 0.09)$ & trace \\
\hline 43 & 3602.8 & 3602.8 & 6 & 5 & 0 & 3 & 0 & & $20.9( \pm 0.26)$ & $24.2( \pm 1.25)$ \\
\hline 44 & 3776.8 & 3776.7 & 6 & 5 & 1 & 3 & 0 & & ND & $2.9( \pm 0.17)$ \\
\hline 45 & 3806.9 & 3806.8 & 6 & 5 & 1 & 2 & 1 & S.1 & $0.1( \pm 0.08)$ & $0.5( \pm 0.07)$ \\
\hline 46 & 3864.9 & 3864.8 & 7 & 6 & 1 & 2 & 0 & 20 & trace & $0.7( \pm 0.06)$ \\
\hline 47 & 3964.0 & 3963.9 & 6 & 5 & 0 & 4 & 0 & $x_{0}=$ & $2.9( \pm 0.11)$ & $3.3( \pm 0.14)$ \\
\hline
\end{tabular}

${ }^{\mathrm{a}}$ Quantitative sialylated and neutral N-glycan percentages measured by MALDI-TOF MS combined with solid-phase permethylation.

${ }^{\mathrm{b}} \mathrm{ND}=$ not detected

${ }^{\mathrm{C}}$ Calculated masses

${ }^{\mathrm{d}}$ Experimental masses

glycans, were previously found in other pig organs (Kim et al., 2006; 2008; 2009a; 2009b). Quantitative MS and HPLC studies of pig glycans showed that this antigen was the most abundant $\alpha$-Gal antigen in WT pig (Kim et al., 2008). We therefore suggested that the $\alpha$-Gal-terminated biantennary glycan with a core fucose $\left(\mathrm{Hex}_{7} \mathrm{HexNAc}_{4} \mathrm{Fuc}_{1}\right)$ might be the only $\alpha$-Gal antigen structure of $\mathrm{N}$-glycans expressed in WT pig fibroblasts. However, the level of this $\alpha$-Gal terminated $N$-glycan was fairly low in the pig fibroblasts used in this study. Recently, Miyagawa et al. (2010) indicated that the level of the $\alpha-G a l$ epitope in pig 
$\boldsymbol{A}$
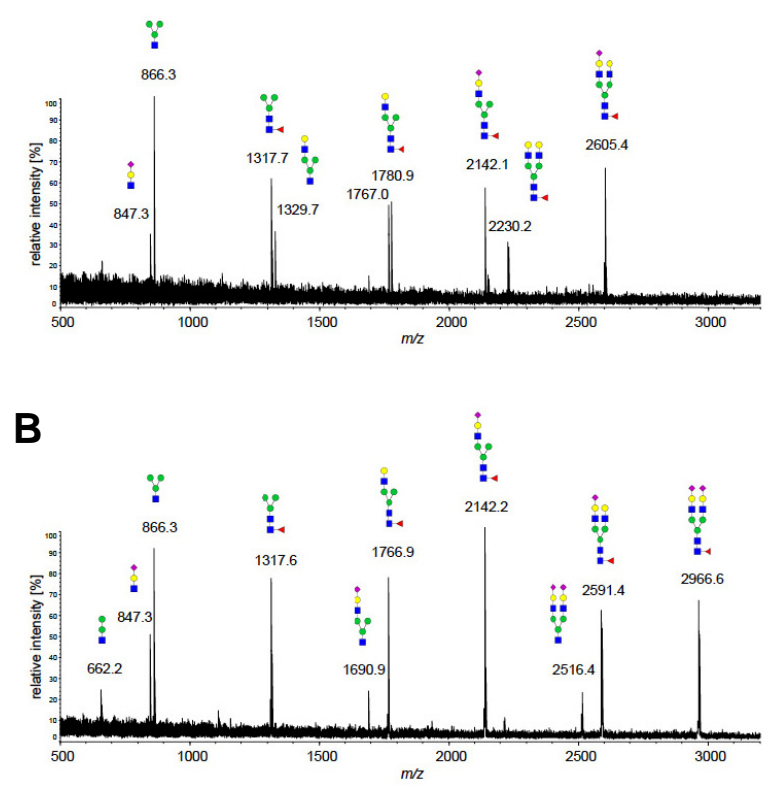
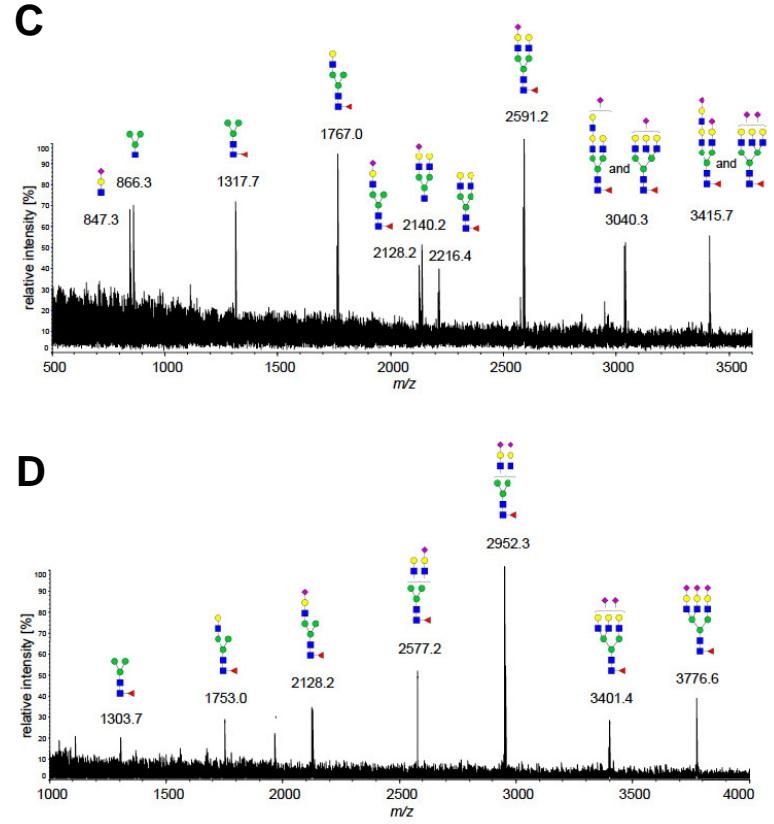

Fig. 5. Positive ion MALDI-QIT-TOF MS/MS spectra of permethylated sialyl glycans observed at $\mathrm{m} / \mathrm{z}$ values of (A) 2605.4, (B) 2966.6, (C) 3415.7, and (D) 3776.6.

A

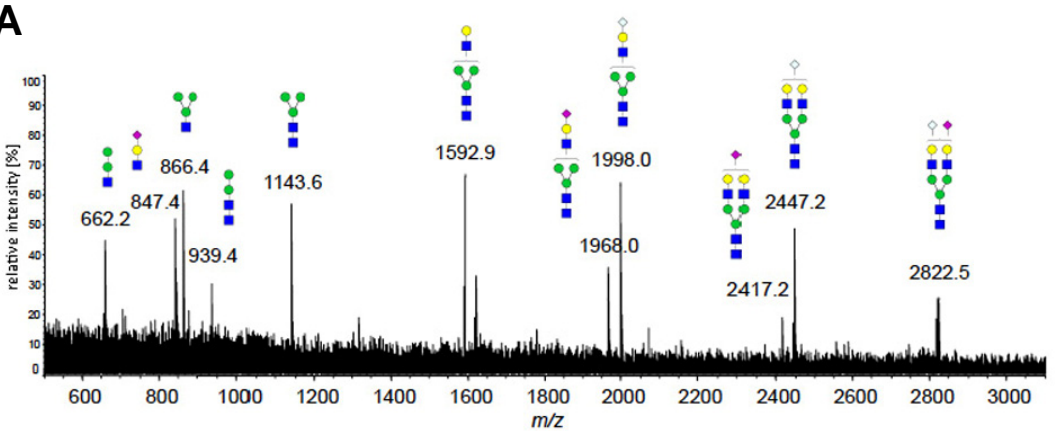

B

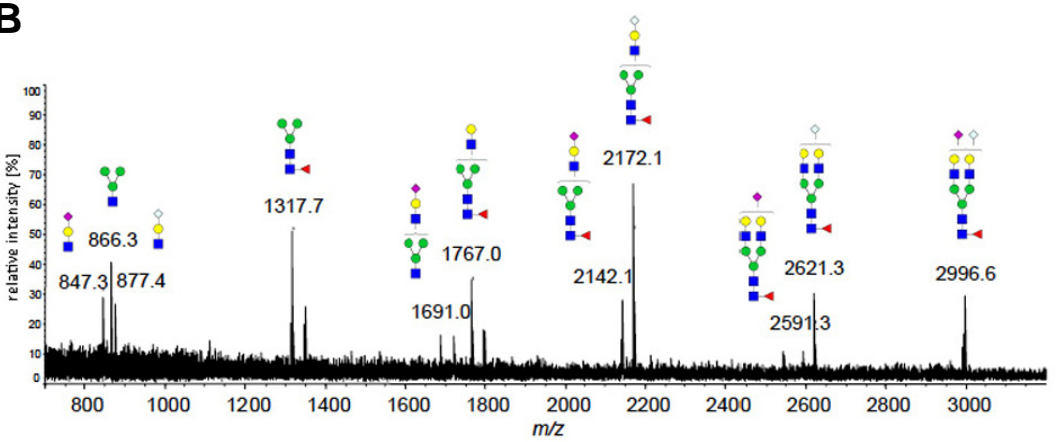

Fig. 6. Positive ion MALDI-QIT-TOF MS/MS spectra of the permethylated NeuGc-containing $\mathrm{N}$-glycans observed at $\mathrm{m} / \mathrm{z}$ (A) 2822.5 and (B) 2996.6 . fibroblast glycolipid is also significantly lower than other glycoepitopes. Thus, this observation may suggest that WT pig fibroblasts express low levels of $\alpha$-Gal antigen compared to other cells/tissues of pigs. However, although $\alpha$-galactosylation does not seem to play a predominant role in fibroblast glycosylation, the disruption of $\alpha$-galactosyltransferase may profoundly influence the expression of other carbohydrate epitopes. This study also indicates that NeuGc is still present on the cell surface of pig fibroblasts after deletion of the $\alpha 1,3$-galactosyl- transferase gene, and thus, the NeuGc antigen may be the next hurdle for further studies in pig-to-human xenotransplantation, as described previously (Morozumi et al., 1999).

For quantitative comparison of $\mathrm{N}$-glycans between WT and GalT-KO pig fibroblasts, MALDI-TOF MS analysis combined with solid-phase permethylation was performed. This approach has been reported to be a good quantitative method with excellent correlation to HPLC for reductive amination derivatization (Wada et al., 2007). Moreover, solid-phase permethylation 
minimizes sialic acid loss via oxidative degradation and peeling. It is therefore more accurate and efficient relative to conventional solution-phase permethylation (Kang et al., 2005). The quantitative result in the present study supports findings by recent studies on different organs of the GalT-KO pig (Park et al., 2011; 2012). Park et al. used an enzyme-linked lectinosorbent assay method to report that $\alpha 1,3-$ galactosyltransferase deficiency in pigs increased sialic acid-terminated glycoconjugate epitopes (Park et al., 2011; 2012). This lectin-based method was able to analyze specific carbohydrate epitopes expressed on the cell surface but did not provide quantitative information on individual glycans or the glycan structures in the GalT-KO pig.

This study also reveals the increase of core-fucosylation levels in the pig GalT-KO through quantitative and structural MS analysis. However, a previous report indicated that there is no significant difference in fucose levels between GalT-KO and WT pig fibroblasts according to the lectin microarray-based glycomics (Miyagawa et al., 2010). In contrast, Diswall et al. reported elevated fucose levels in glycolipids from GalT-KO pig tissues and structurally identified them with LC-MS and proton NMR (Diswall et al., 2010). Thus, the potential role of the glycosyltransferases in GalT-KO pig remains to be elucidated.

The detection and quantitative measurement of NeuGc could not be carried out in previous studies because there was no available commercial lectin that specifically binds to the NeuGc epitope (Miyagawa et al., 2010; 2013). Recently, Park et al. reported that quantification of NeuGc was performed via 1,2diamino-4,5-methylenedioxybenzene (DMB) derivatization of sialic acids. However, they did not distinguish between the major glycan types, such as $\mathrm{N}$ - and $\mathrm{O}$-glycans and glycolipidderived glycans (Park et al., 2012). Here, we performed $N$ glycan-specific analysis using PNGase F enzyme digestion, and thus we were able to use MS analysis to obtain more detailed information on the NeuGc-terminated $\mathrm{N}$-glycan structure. Although the relative change in NeuGc quantity between WT and GalT-KO might not seem dramatic, the increase of the NeuGc antigen in the fibroblasts matched previous results on GalT-KO pig organs (Park et al., 2011; 2012) indicating that the heart, liver, and kidneys of GalT-KO pigs had higher levels of NeuGc than did those of WT pigs. Two NeuGc-linked N-glycan structures from fibroblasts were investigated in this study. Unfortunately, it was difficult to assign the other $N$-glycans, which were expected to contain NeuGc residues, because their peak intensities were too low to be isolated in MS/MS. Nevertheless, we showed here that the MS-based approach enabled qualitative and quantitative analysis of cellular $\mathrm{N}$-glycans, including xenoantigens from WT and GalT-KO pig cells. Taken together, we concluded that sialic acid-containing glycoconjugates with NeuGc antigen expression in pigs increased following deletion of the $\alpha$ 1,3-galactosyltransferase gene.

In summary, a total of $47 \mathrm{~N}$-glycans isolated from WT and GalT-KO pig fibroblasts were identified using an MS approach. We illustrated the relative change in $\mathrm{N}$-glycosylation including NeuGc between WT and GalT-KO, suggesting that deletion of the $\alpha 1,3$-galactosyltransferase gene may not only remove the construction of the Gal $1,3 \mathrm{Gal}$ residue, but may also increase the amount of sialylated glycans, including the NeuGc antigen. Therefore, this study provides important information for further research of pre-clinical trials involving GalT-KO pigs.

\section{ACKNOWLEDGMENTS}

This work was supported by Basic Science Research Program through the National Research Foundation of Korea (NRF) (NRF-2013R1A1A1004998), Polar Academic Program (PAP,
PD14010) from KOPRI and a Korea Basic Science Institute grant (T34617).

\section{REFERENCES}

Ahn, Y., Kang, U.B., Kim, J., and Lee, C. (2010). Mining of serum glycoproteins by an indirect approach using cell line secretome. Mol. Cells 29, 123-130

Ahn, K.S., Kim, Y.J., Kim, M., Lee, B.H., Heo, S.Y., Kang, M.J., Kang, Y.K., Lee, J.W., Lee, K.K., Kim, J.H., et al. (2011). Resurrection of an alpha-1,3-galactosyltransferase gene-targeted miniature pig by recloning using postmortem ear skin fibroblasts. Theriogenology 75, 933-939.

Baumann, B.C., Stussi, G., Huggel, K., Rieben, R., and Seebach J.D. (2007). Reactivity of human natural antibodies to endothelial cells from Galalpha $(1,3)$ Gal-deficient pigs. Transplantation 83 , 193-201.

Burlak, C., Bern, M., Brito, A.E., Isailovic, D., Wang, Z.Y., Estrada, J.L., Li, P., and Tector, A.J. (2013). N-linked glycan profiling of GGTA1/CMAH knockout pigs identifies new potential carbohydrate xenoantigens. Xenotransplantation 20, 277-291.

Crocker, P.R., and Feizi, T. (1996). Carbohydrate recognition systems: functional triads in cell-cell interactions. Curr. Opin. Struct. Biol. 6, 679-691

Dai, Y., Vaught, T.D., Boone, J., Chen, S.H., Phelps, C.J., Ball, S., Monahan, J.A., Jobst, P.M., McCreath, K.J., Lamborn, A.E., et al. (2002). Targeted disruption of the alpha1,3-galactosyltransferase gene in cloned pigs. Nat. Biotechnol. 20, 251-255.

Diswall, M., Angstrom, J., Karlsson, H., Phelps, C.J., Ayares, D., Teneberg, S., and Breimer, M.E. (2010). Structural characterization of alpha1,3-galactosyltransferase knockout pig heart and kidney glycolipids and their reactivity with human and baboon antibodies. Xenotransplantation 17, 48-60.

Dube, D.H., and Bertozzi, C.R. (2005). Glycans in cancer and inflammation--potential for therapeutics and diagnostics. Nat. Rev. Drug Discov. 4, 477-488.

Fujimura, T., Takahagi, Y., Shigehisa, T., Nagashima, H., Miyagawa, S., Shirakura, R., and Murakami, H. (2008). Production of alpha 1,3-galactosyltransferase gene-deficient pigs by somatic cell nuclear transfer: a novel selection method for gal alpha 1,3-Gal antigen-deficient cells. Mol. Rep. Dev. 75, 1372-1378.

Gil, G.C., Kim, Y.G., and Kim, B.G. (2008). A relative and absolute quantification of neutral $N$-linked oligosaccharides using modification with carboxymethyl trimethylammonium hydrazide and matrix-assisted laser desorption/ionization time-of-flight mass spectrometry. Anal. Biochem. 379, 45-59.

Gil, G.C., lliff, B., Cerny, R., Velander, W.H., and Van Cott, K.E. (2010). High throughput quantification of $\mathrm{N}$-glycans using onepot sialic acid modification and matrix assisted laser desorption ionization time-of-flight mass spectrometry. Anal. Chem. 82, 6613-6620.

Haslam, S.M., North, S.J., and Dell, A. (2006). Mass spectrometric analysis of $\mathrm{N}$ - and O-glycosylation of tissues and cells. Curr. Opin. Struct. Biol. 16, 584-591.

Jang, K.S., Kim, Y.G., Gil, G.C., Park, S.H., and Kim, B.G. (2009). Mass spectrometric quantification of neutral and sialylated $N$ glycans from a recombinant therapeutic glycoprotein produced in the two Chinese hamster ovary cell lines. Anal. Biochem. 386, 228-236.

Kang, P., Mechref, Y., Klouckova, I., and Novotny, M.V. (2005). Solid-phase permethylation of glycans for mass spectrometric analysis. Rapid Commun. Mass Spectrom. 19, 3421-3428.

Kang, P., Mechref, Y., and Novotny, M.V. (2008). High-throughput solid-phase permethylation of glycans prior to mass spectrometry. Rapid Commun. Mass Spectrom. 22, 721-734.

Kim, Y.G., Kim, S.Y., Hur, Y.M., Joo, H.S., Chung, J., Lee, D.S Royle, L., Rudd, P.M., Dwek, R.A., Harvey, D.J., et al. (2006). The identification and characterization of xenoantigenic nonhuman carbohydrate sequences in membrane proteins from porcine kidney. Proteomics 6, 1133-1142.

Kim, Y.G., Gil, G.C., Harvey, D.J., and Kim, B.G. (2008). Structural analysis of alpha-Gal and new non-Gal carbohydrate epitopes from specific pathogen-free miniature pig kidney. Proteomics 8 , 2596-2610.

Kim, Y.G., Gil, G.C., Jang, K.S., Lee, S., Kim, H.I., Kim, J.S., Chung, 
J., Park, C.G., Harvey, D.J., and Kim, B.G. (2009a). Qualitative and quantitative comparison of $\mathrm{N}$-glycans between pig endothelial and islet cells by high-performance liquid chromatography and mass spectrometry-based strategy. J. Mass Spectrom. 44, 1087-1104.

Kim, Y.G., Oh, J.Y., Gil, G.C., Kim, M.K., Ko, J.H., Lee, S., Lee, H.J., Wee, W.R., and Kim, B.G. (2009b). Identification of alpha-Gal and non-Gal epitopes in pig corneal endothelial cells and keratocytes by using mass spectrometry. Curr. Eye Res. 34, 877-895.

Kolber-Simonds, D., Lai, L., Watt, S.R., Denaro, M., Arn, S., Augenstein, M.L., Betthauser, J., Carter, D.B., Greenstein, J.L., Hao, Y., et al. (2004). Production of alpha-1,3-galactosyltransferase null pigs by means of nuclear transfer with fibroblasts bearing loss of heterozygosity mutations. Proc. Natl. Acad. Sci. USA 101, 7335-7340.

Lai, L., Kolber-Simonds, D., Park, K.W., Cheong, H.T., Greenstein, J.L., Im, G.S., Samuel, M., Bonk, A., Rieke, A., Day, B.N., et al (2002). Production of alpha-1,3-galactosyltransferase knockout pigs by nuclear transfer cloning. Science 295, 1089-1092.

Lutz, A.J., Li, P., Estrada, J.L., Sidner, R.A., Chihara, R.K., Downey, S.M., Burlak, C., Wang, Z.Y., Reyes, L.M., Ivary, B., et al. (2013). Double knockout pigs deficient in $\mathrm{N}$-glycolylneuraminic acid and galactose alpha-1,3-galactose reduce the humoral barrier to xenotransplantation. Xenotransplantation 20, 27-35.

Marth, J.D., and Grewal, P.K. (2008). Mammalian glycosylation in immunity. Nat. Rev. Immunol. 8, 874-887.

Miyagawa, S., Takeishi, S., Yamamoto, A., Ikeda, K., Matsunari, H., Yamada, M., Okabe, M., Miyoshi, E., Fukuzawa, M., and Nagashima, H. (2010). Survey of glycoantigens in cells from alpha1-3galactosyltransferase knockout pig using a lectin microarray. Xenotransplantation 17,61-70.

Miyagawa, S., Maeda, A., Takeishi, S., Ueno, T., Usui, N., Matsumoto, S., Okitsu, T., Goto, M., and Nagashima, H.A (2013) lectin array analysis for wild-type and alpha-Gal-knockout pig islets versus healthy human islets. Surg. Today 43, 1439-1447.

Morozumi, K., Kobayashi, T., Usami, T., Oikawa, T., Ohtsuka, Y., Kato, M., Takeuchi, O., Koyama, K., Matsuda, H., Yokoyama, I., et al. (1999). Significance of histochemical expression of Hanganutziu-Deicher antigens in pig, baboon and human tissues. Transplant Proc. 31, 942-944.

North, S.J., Hitchen, P.G., Haslam, S.M., and Dell, A. (2009). Mass spectrometry in the analysis of $\mathrm{N}$-linked and O-linked glycans. Curr. Opin. Struct. Biol. 19, 498-506.

Ohtsubo, K., and Marth, J.D. (2006). Glycosylation in cellular mechanisms of health and disease. Cell 126, 855-867.

Park, J.Y., Park, M.R., Kwon, D.N., Kang, M.H., Oh, M., Han, J.W., Cho, S.G., Park, C., Kim, D.K., Song, H., et al. (2011). Alpha 1,3galactosyltransferase deficiency in pigs increases sialyltransferase activities that potentially raise non-gal xenoantigenicity. J. Biomed. Biotechnol. 2011, 560850

Park, J.Y., Park, M.R., Bui, H.T., Kwon, D.N., Kang, M.H., Oh, M., Han, J.W., Cho, S.G., Park, C., Shim, H., et al. (2012). alpha1,3galactosyltransferase deficiency in germ-free miniature pigs increases $\mathrm{N}$-glycolylneuraminic acids as the xenoantigenic determinant in pig-human xenotransplantation. Cell Reprogram 14 353-363.

Puga Yung, G.L., Li, Y., Borsig, L., Millard, A.L., Karpova, M.B. Zhou, D., and Seebach, J.D. (2012) Complete absence of the alphaGal xenoantigen and isoglobotrihexosylceramide in alpha1,3galactosyltransferase knock-out pigs. Xenotransplantation 19, 196-206.

Roseman, S. (2001). Reflections on glycobiology. J. Biol. Chem. 276, 41527-41542.

Roth, J., Zuber, C., Park, S., Jang, I., Lee, Y., Kysela, K. G., Le Fourn, V., Santimaria, R., Guhl, B., and Cho, J.W. (2010). Protein $\mathrm{N}$-glycosylation, protein folding, and protein quality control. Mol. Cells 30, 497-506.

Sandrin, M.S., and McKenzie, I.F. (1994). Gal alpha $(1,3)$ Gal, the major xenoantigen(s) recognised in pigs by human natural antibodies. Immunol. Rev. 141, 169-190.

Toyoda, M., Ito, H., Matsuno, Y.K., Narimatsu, H., and Kameyama, A. (2008). Quantitative derivatization of sialic acids for the detection of sialoglycans by MALDI MS. Anal. Chem. 80, 5211-5218.

Wada, Y., Azadi, P., Costello, C.E., Dell, A., Dwek, R.A., Geyer, H., Geyer, R., Kakehi, K., Karlsson, N.G., Kato, K., et al. (2007) Comparison of the methods for profiling glycoprotein glycans-HUPO human disease glycomics/proteome initiative multiinstitutional study. Glycobiology 17, 411-422.

Zhao, Y.Y., Takahashi, M., Gu, J.G., Miyoshi, E., Matsumoto, A., Kitazume, S., and Taniguchi, N. (2008). Functional roles of $N$ glycans in cell signaling and cell adhesion in cancer. Cancer Sci. 99, 1304-1310. 